\title{
BMJ Open Are medical record front page data suitable for risk adjustment in hospital performance measurement? Development and validation of a risk model of in-hospital mortality after acute myocardial infarction
}

Chaoqun Wu, ${ }^{1}$ Danwei Zhang, ${ }^{1}$ Xueke Bai, ${ }^{1}$ Tiannan Zhou, ${ }^{2}$ Yongfei Wang, ${ }^{3}$ Zhenqiu Lin, ${ }^{2}$ Guangda He, ${ }^{1}$ Xi Li (1) , ${ }^{1}$ for the China PEACE Collaborative Group

To cite: Wu C, Zhang D, Bai X, et al. Are medical record front page data suitable for risk adjustment in hospital performance measurement? Development and validation of a risk model of in-hospital mortality after acute myocardial infarction. BMJ Open 2021;11:e045053. doi:10.1136/ bmjopen-2020-045053

- Prepublication history and supplemental materials for this paper is available online. To view these files, please visit the journal online (http://dx.doi. org/10.1136/bmjopen-2020045053).

Received 22 September 2020 Revised 25 January 2021 Accepted 16 March 2021
(D) Check for updates

\section{(C) Author(s) (or their} employer(s)) 2021. Re-use permitted under CC BY-NC. No commercial re-use. See rights and permissions. Published by BMJ.

For numbered affiliations see end of article.

Correspondence to Dr Xi Li; xi.li@fwoxford.org

\section{ABSTRACT}

Objectives To develop a model of in-hospital mortality using medical record front page (MRFP) data and assess its validity in case-mix standardisation by comparison with a model developed using the complete medical record data. Design A nationally representative retrospective study. Setting Representative hospitals in China, covering 161 hospitals in modelling cohort and 156 hospitals in validation cohort.

Participants Representative patients admitted for acute myocardial infarction. 8370 patients in modelling cohort and 9704 patients in validation cohort.

Primary outcome measures In-hospital mortality, which was defined explicitly as death that occurred during hospitalisation, and the hospital-level risk standardised mortality rate (RSMR).

Results A total of 14 variables were included in the model predicting in-hospital mortality based on MRFP data, with the area under receiver operating characteristic curve of 0.78 among modelling cohort and 0.79 among validation cohort. The median of absolute difference between the hospital RSMR predicted by hierarchical generalised linear models established based on MRFP data and complete medical record data, which was built as 'reference model', was $0.08 \%$ (10th and 90th percentiles: $-1.8 \%$ and $1.6 \%$ ). In the regression model comparing the RSMR between two models, the slope and intercept of the regression equation is 0.90 and 0.007 in modelling cohort, while 0.85 and 0.010 in validation cohort, which indicated that the evaluation capability from two models were very similar.

Conclusions The models based on MRFP data showed good discrimination and calibration capability, as well as similar risk prediction effect in comparison with the model based on complete medical record data, which proved that MRFP data could be suitable for risk adjustment in hospital performance measurement.

\section{INTRODUCTION}

Equal access to high-quality healthcare is one of the major aims in China's recent public
Strengths and limitations of this study

- The analysis was based on a nationally representative cohort of hospitals in China, from which random samples of patients admitted with acute myocardial infarction was drawn to represent the heterogeneity in outcome of care.

- We used hierarchical generalised linear models that fully considered the patient clustering in hospitals and were able to distinguish the differences within and between hospitals, which suits the purpose to adjust for case-mix in hospital performance comparison.

- We validated the finding that concise data extracted from medical record front page are good enough to reflect patients' risk profile using the data from a closer year.

- External validations that include more diverse hospitals and among other diseases will be needed in the future.

hospital reform. ${ }^{12}$ To continuously improve quality of care and mitigate its disparities across regions or hospitals, sustainable monitoring of hospital performance, particularly patient outcomes, is first required. ${ }^{3}{ }^{4}$ The Ministry of Health (named as 'National Health Commission' now) of China established the Hospital Quality Monitoring System (HQMS) in 2011 to collect key information of all hospitalisations, including patients' diagnosis and outcomes recorded in the medical record front page (MRFP) using a standardised form (online supplemental table S1). ${ }^{56}$ Although the MRFP lack of detailed information on treatment process such as lab test results or medications, with structured records on diagnosis, procedure and outcome, it could 
be used as a unique nationwide data source of outcome quality assessment (ie, in-hospital mortality).

Assessing quality of care between hospitals needs to take into account patients' different demographic and clinical characteristics of patients between hospitals, like most of the prior studies have done based on a broad array of information from complete medical record. ${ }^{7-9}$ However, it is still unclear whether the MRFP data collected in HQMS can act as good surrogates for complete medical record model in estimation of risk-standardised mortality.

In China Patient-centred Evaluative Assessment of Cardiac Events (PEACE) retrospective study, we built a nationally representative sample of patients hospitalised for acute myocardial infarction (AMI) and extracted high-quality data from their complete medical records (including MRFPs), which provided an ideal condition to assess disparities in quality of care. ${ }^{10}$ We aim to develop a model of in-hospital mortality using their MRFP data, then assess its effect in case-mix standardisation by comparing with a model developed using the complete medical record data of the same patient cohort.

\section{METHODS}

Patient and public involvement

No patient involved.

\section{Study design and population}

The design of China PEACE-Retrospective AMI study has been published previously. ${ }^{11}$ In brief, the study used a stratified two-stage random sampling method to select representative hospitals and patients admitted for AMI nationwide during 2001, 2006 and 2011. In addition, the study also included a more recent sample of patients admitted in 2015 using the same random sampling process. First, five regions (Eastern cities, Central and Western cities, Eastern villages, Central villages and Western villages) were used for representative hospital selection by simple random sampling method. Second, AMI cases (identified accoding to International Classification of Diseases-Clinical Modification codes, versions 9 (410.xx) and 10 (I21.xx), or key words from discharge diagnosis) were randomly selected from all patients who met the inclusion criteria in each selected hospital by random sampling method. Trained personnel at the national coordinating centres abstracted data from the medical records using standardised data definitions. Data abstraction quality was monitored by random audits that ensured that the overall variable accuracy exceeded $98 \% .^{11}$

As a retrospective study, written informed consent of patients were not required.

In this study, patients from year 2011 were regarded as the modelling cohort, and patients from the year 2015 were regarded as the validation cohort. Patients who transferred out to another hospital were excluded since we could not get their outcomes. A total of 8370 patients from 161 hospitals (96 secondary hospitals and 65 tertiary hospitals) were included as modelling cohort, and another 9704 patients from 156 hospitals (93 secondary hospitals and 63 tertiary hospitals) were included as validation cohort. In addition, if a hospital had less than 10 eligible patients per year, it would be further excluded from the hospital-level analysis. A total of 8269 patients (137 hospitals, 73 secondary hospitals and 64 tertiary hospitals) from modelling cohort and 9583 patients (132 hospitals, 71 secondary hospitals and 61 tertiary hospitals) from validation cohort were included in the further analysis (online supplemental figure S1).

\section{Statistical analysis}

According to study aim, we need to develop and evaluate a model predicting in-hospital outcome at patient level based on MRFP data from modelling cohort first. If the model performed well, then another model used to evaluate hospital quality of care would be built based on prior model. The validation cohort was used to conduct external evaluation of models. Hospital-level model would be built based on complete medical record data, which could be considered as 'the best reference'. By comparing the difference and association of the indicators evaluated by the MRFP model and the complete medical record model, we could explore whether the model based on MRFP data had similar efficiency with that based on complete medical record data. The analysis roadmap was demonstrated in figure 1.

\section{Candidate predictors and outcome}

Patient characteristics were selected as candidate predictors, according to previous AMI predictive models such as GRACE, TIMI and ACTION-GWTG. ${ }^{7-9}{ }^{12-17}$ For the model based on MRFP data, the candidate predictors included demographic characteristics (gender, age, medical insurance status, ethnicity and marital status), admission department, diagnosis at admission (cardiac arrest) and at discharge (acute ST-segment elevation myocardial infarction (STEMI), infarction position, hypertension, diabetes, dyslipidaemia, cardiogenic shock, heart failure, stroke and renal failure), which was available from MRFP data. For the model based on complete medical record, we additionally include patients' symptoms, vital signs and lab test results at admission.

In-hospital mortality, as the outcome variable in the models, was defined explicitly as death that occurred during hospitalisation, which was recorded both on the MRFP and elsewhere such as discharge record. For the accuracy of analysis, we used complete medical record as data source. We did not include patients who withdraw treatment as outcome since we could not get 'withdraw' information from MRFP data, though plenty of these patients might die soon after giving up treatment.

\section{Patient-level model development and evaluation}

A logistic regression model was built based on MRFP data from the modelling cohort. Area under receiver operating characteristic curve (AUC) and observed rates in deciles 
Select candidate variables

-clinical knowledge, article summary, backward method of logistic model

\section{Evaluating stability of model 1}

-apply select covariates to validation cohort and reestimate logistic model

-discrimination and calibration ability

Fit HGLM model(model 4)

-using selected covariates, hospital level random effect -achieve the estimated RSMR of validation cohort
Select candidate variables

-clinical knowledge, article summary, backward method of logistic model

Model evaluation

discrimination and calibration ability

Fit HGLM model(model 3) -using selected covariates, hospital level random effect

achieve the RSMR of modelling cohort

Evaluating HGLM result -absolute differences between model 2 and model 3

regression model using RSMR from model 3 as dependent variable and RSMR from model 2 as the independent variable

Fit HGLM model(model 5)

-using selected covariates, hos pital level as random effect achieve the RSMR of validation cohort

Evaluating HGLM result -absolute differences between model 4 and model 5

regression model using RSMR from model 5 as dependent variable and RSMR from model 4 as the independent variable

Figure 1 Analysis roadmap. HGLM, hierarchical generalised linear model; RSMR, risk standardised mortality rate.

determined by model estimating value were used to evaluate the discrimination. Slope and intercept of regression equation between the observed and the predicted mortality was used to evaluate the calibration ability. To assess the overfitting of the model, we used the coefficients estimated from the logistic model to predict the probability of mortality in the validation cohort by multiplying coefficients by the observed risk factors variables 
and summing over for each subject. Then another logistic regression model was built, in which the dependent variable was observed mortality and independent variables were the predicted mortality generated as above. The slope different from 1 and the intercept different from 0 indicated overfitting.

Furthermore, we re-estimated the logistic regression model in the validation cohort used selected predictors above. If the estimated coefficients of new model were similar to prior, the selected predictors were considered to be stable. Discrimination and calibration were also evaluated in the re-established logistic model.

Complete medical record model was developed and validated based on the data from complete medical records, using the same method mentioned above. Additionally, we compared the performance of our complete medical record model and MRFP model with the GRACE in-hospital mortality $\operatorname{model}^{7}$ among development and validation cohorts by calculating the difference of AUC and the integrated discrimination improvement (IDI) (online supplemental appendix A).

\section{Hospital-level model development and comparison}

Hierarchical generalised linear models (HGLMs) were established among modelling and validation cohort separately using above selected covariates and hospitals as random effects. HGLM considered the patient clustering in hospitals and could be used to distinguish the differences of outcome within and between hospitals.

Hospital-level risk standardised mortality rate (RSMR) was used as an indicator to evaluating hospital quality of care in this study. The RSMR of each hospital could be calculated from HGLM as the ratio of predicted and expected mortality of the hospital, multiplied by the unadjusted rate of all hospitals. The expected mortality is the mortality rate of the hospital if patients in each hospital were treated in a 'reference' hospital; the predicted mortality accounted for the characteristics of a hospital (the hospital-level random effects of the model). ${ }^{818}$

We use two methods to compare the RSMR derived from the HGLMs based on MRFP and the complete medical record data: (1) absolute differences of RSMR from two models were calculated, and the distribution of differences was described using mean, median and maximum. (2) A linear regression model was built, with RSMR from the complete medical record data as the dependent variable and RSMR from the MRFP data as the independent variable. The slope of the model approaching 1 and the intercept approaching 0 indicated that the predicted probabilities from the two models were very similar. All above calculation and comparison would be conducted among the modelling and validation cohort separately.

All statistical inferences were performed on two-tailed test, and $\mathrm{p}<0.05$ was considered statistically significant.
Table 1 Patients' characteristics from MRFP data and inhospital mortality in modelling cohort and validation cohort

\begin{tabular}{|c|c|c|c|}
\hline & $\begin{array}{l}\text { Modelling } \\
\text { cohort (year } \\
\text { 2011) }\end{array}$ & $\begin{array}{l}\text { Validation } \\
\text { cohort (year } \\
\text { 2015) }\end{array}$ & \\
\hline & $\mathrm{N}=8370$ & $\mathrm{~N}=9704$ & $P$ value \\
\hline In-hospital mortality & $621(7.4)$ & $687(7.1)$ & 0.3793 \\
\hline Female & $2519(30.1)$ & $3121(32.2)$ & 0.0028 \\
\hline Age (years), mean (SD) & $65.4(12.8)$ & $65.9(12.7)$ & 0.0081 \\
\hline$<40$ & $195(2.3)$ & $213(2.2)$ & $<0.0001$ \\
\hline $40-49$ & $910(10.9)$ & $891(9.2)$ & \\
\hline $50-59$ & $1600(19.1)$ & $1816(18.7)$ & \\
\hline $60-69$ & $2090(25.0)$ & $2674(27.6)$ & \\
\hline $70-79$ & $2431(29.0)$ & $2590(26.7)$ & \\
\hline$\geq 80$ & $1144(13.7)$ & $1520(15.7)$ & \\
\hline Han & $7701(92.0)$ & $9285(95.7)$ & $<0.0001$ \\
\hline Married & $7460(89.1)$ & $8740(90.1)$ & 0.0391 \\
\hline Having medical insurance & $5126(61.2)$ & $7507(77.4)$ & $<0.0001$ \\
\hline $\begin{array}{l}\text { Admission at } \\
\text { cardiology department }\end{array}$ & $4087(48.8)$ & $6532(67.3)$ & $<0.0001$ \\
\hline \multicolumn{4}{|l|}{ Admission diagnosis } \\
\hline Cardiac arrest & $6(0.1)$ & $18(0.2)$ & 0.0362 \\
\hline \multicolumn{4}{|l|}{ Discharge diagnosis } \\
\hline STEMI & $5509(65.8)$ & $4753(49.0)$ & $<0.0001$ \\
\hline $\begin{array}{l}\text { Acute extensive } \\
\text { anterior } \mathrm{Ml}\end{array}$ & 967 (11.6) & $769(7.9)$ & $<0.0001$ \\
\hline Acute anterior $\mathrm{MI}$ & $1504(18.0)$ & $1310(13.5)$ & $<0.0001$ \\
\hline $\begin{array}{l}\text { Acute anterior septal } \\
\text { Ml }\end{array}$ & $587(7.0)$ & $408(4.2)$ & $<0.0001$ \\
\hline Acute inferior $\mathrm{Ml}$ & $2558(30.6)$ & $2214(22.8)$ & $<0.0001$ \\
\hline Acute lateral MI & $359(4.3)$ & $311(3.2)$ & 0.0001 \\
\hline Acute posterior MI & $699(8.4)$ & $502(5.2)$ & $<0.0001$ \\
\hline $\begin{array}{l}\text { Acute right ventricular } \\
\text { infarction }\end{array}$ & $615(7.3)$ & $418(4.3)$ & $<0.0001$ \\
\hline Hypertension & $3894(46.5)$ & $5080(52.3)$ & $<0.0001$ \\
\hline Diabetes mellitus & $1650(19.7)$ & $2345(24.2)$ & $<0.0001$ \\
\hline Dyslipidaemia & $836(10.0)$ & $1434(14.8)$ & $<0.0001$ \\
\hline Cardiogenic shock & $403(4.8)$ & $510(5.3)$ & 0.1773 \\
\hline Heart failure & $2853(34.1)$ & $3793(39.1)$ & $<0.0001$ \\
\hline Stroke & $655(7.8)$ & $1389(14.3)$ & $<0.0001$ \\
\hline Renal failure & $259(3.1)$ & $684(7.0)$ & $<0.0001$ \\
\hline
\end{tabular}

MI, myocardial infarction; MRFP, medical record front page; STEMI, STsegment elevation myocardial infarction.

The statistical software used is SAS V.9.4 (SAS Institute Inc, Cary, North Carolina, USA).

\section{RESULTS}

\section{Study population and characteristics}

In the modelling cohort, the average age was $65.4 \pm 12.8$ years, and $2519(30.1 \%)$ patients were female. About half of the patients were admitted to cardiovascular department at admission. A percentage of $65.8 \%$ were diagnosed with STEMI, while $46.5 \%, 19.7 \%$ and $10.0 \%$ 


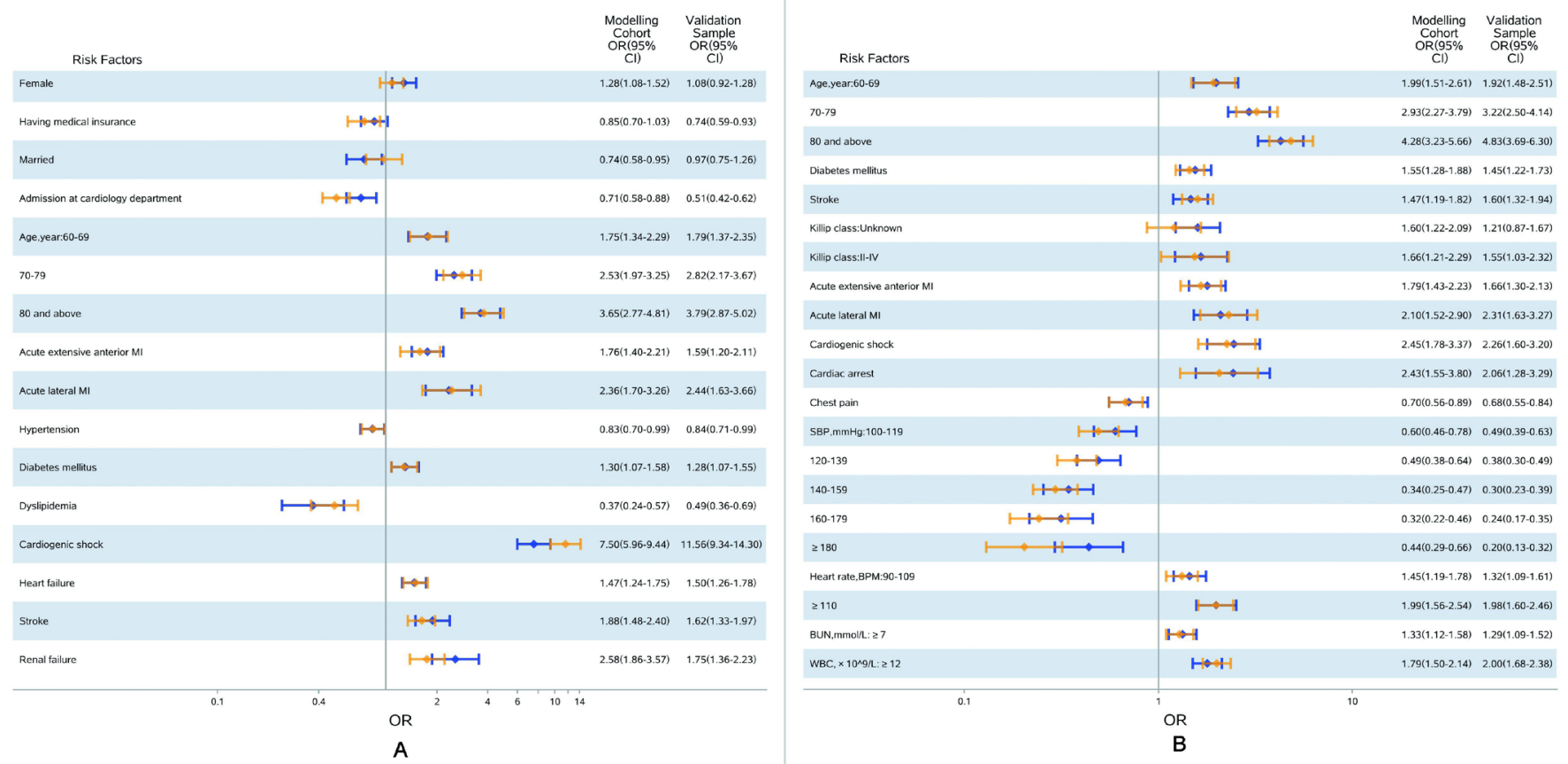

Figure 2 Odds ratios (ORs) of MRFP model and complete medical record model based on modelling and validation cohorts. (A) MRFP model. Note: blue for modelling sample, orange for validation sample. Estimate of between-hospital variance: 0.592 $(\mathrm{SE}=0.125)$ in derivation sample and $0.773(\mathrm{SE}=0.147)$ in variation sample. (B) Complete medical record model. Note: blue for derivation, orange for validation sample. Estimate of between-hospital variance: $0.558(\mathrm{SE}=0.121)$ in derivation sample and 0.719 (SE=0.139) in validation sample. MI, myocardial infarction; MRFP, medical record front page.

had comorbidities of hypertension, diabetes and dyslipidaemia, respectively. Cardiogenic shock occurred in $4.8 \%$ of the patients, and $0.1 \%$ of patients had cardiac arrest before admission (table 1). A total of 621 patients died during hospitalisation, accounting for $7.4 \%$ of the modelling cohort.

Compared with modelling cohort, patients in the validation cohort had a higher proportion of patients with medical insurance and admission in cardiovascular departments $(p<0.001)$. Less proportion $(49.0 \%)$ of patients were diagnosed with STEMI $(p<0.001)$, while a greater proportion of patients had hypertension, diabetes, dyslipidaemia, heart failure and renal failure $(p<0.05)$ (table 1). Six hundred and eighty-nine patients died during hospitalisation, accounting for $7.1 \%$ of the validation cohort, which was not significantly different from the modelling cohort $(\mathrm{p}=0.41)$.

\section{Development and validation of patient-level model}

A total of 14 risk factors were included in the MRFP model based on modelling cohort (figure 2A). Model discrimination was good, with the AUC of 0.78 , and observed mortality rate ranging from $0.83 \%$ in the lowest decile of the predicted mortality rate to $26.88 \%$ in the highest decile. The slope of the calibration curve was 0.91 , and the intercept was -0.007 , which showed the good calibration ability of this model (table 2). The overfitting statistics were within an acceptable range (slope $=1.01$, intercept $=-0.07$ ), indicating that no overfitting exist.

Table 2 Performance of the MRFP model and the complete medical record model

\begin{tabular}{|c|c|c|c|c|}
\hline \multirow[b]{2}{*}{ Model } & \multirow[b]{2}{*}{$\mathbf{N}$} & \multicolumn{2}{|l|}{ Discrimination } & \multirow{2}{*}{$\begin{array}{l}\text { Calibration } \\
\text { Calibration indices } \\
\text { (slope, intercept) }\end{array}$} \\
\hline & & $\begin{array}{l}\text { Area under ROC } \\
\text { curve }\end{array}$ & $\begin{array}{l}\text { Predictive ability* (mean rate } \\
\text { of lowest/highest decile) }\end{array}$ & \\
\hline \multicolumn{5}{|l|}{ MRFP model } \\
\hline Year 2011 (modelling cohort) & 8370 & 0.776 & $0.83 \%-26.88 \%$ & $(0.909,0.007)$ \\
\hline Year 2015 (validation cohort) & 9704 & 0.794 & $1.00 \%-29.72 \%$ & $(0.933,0.005)$ \\
\hline \multicolumn{5}{|l|}{ Complete medical record model } \\
\hline Year 2011 (modelling cohort) & 8370 & 0.790 & $0.51 \%-27.96 \%$ & $(0.940,0.004)$ \\
\hline Year 2015 (validation cohort) & 9704 & 0.798 & $0.92 \%-28.69 \%$ & $(0.927,0.005)$ \\
\hline
\end{tabular}

${ }^{*}$ Observed rates in deciles determined by estimated model.

$\mathrm{ROC}$, receiver operating characteristic; MRFP, medical record front page. 

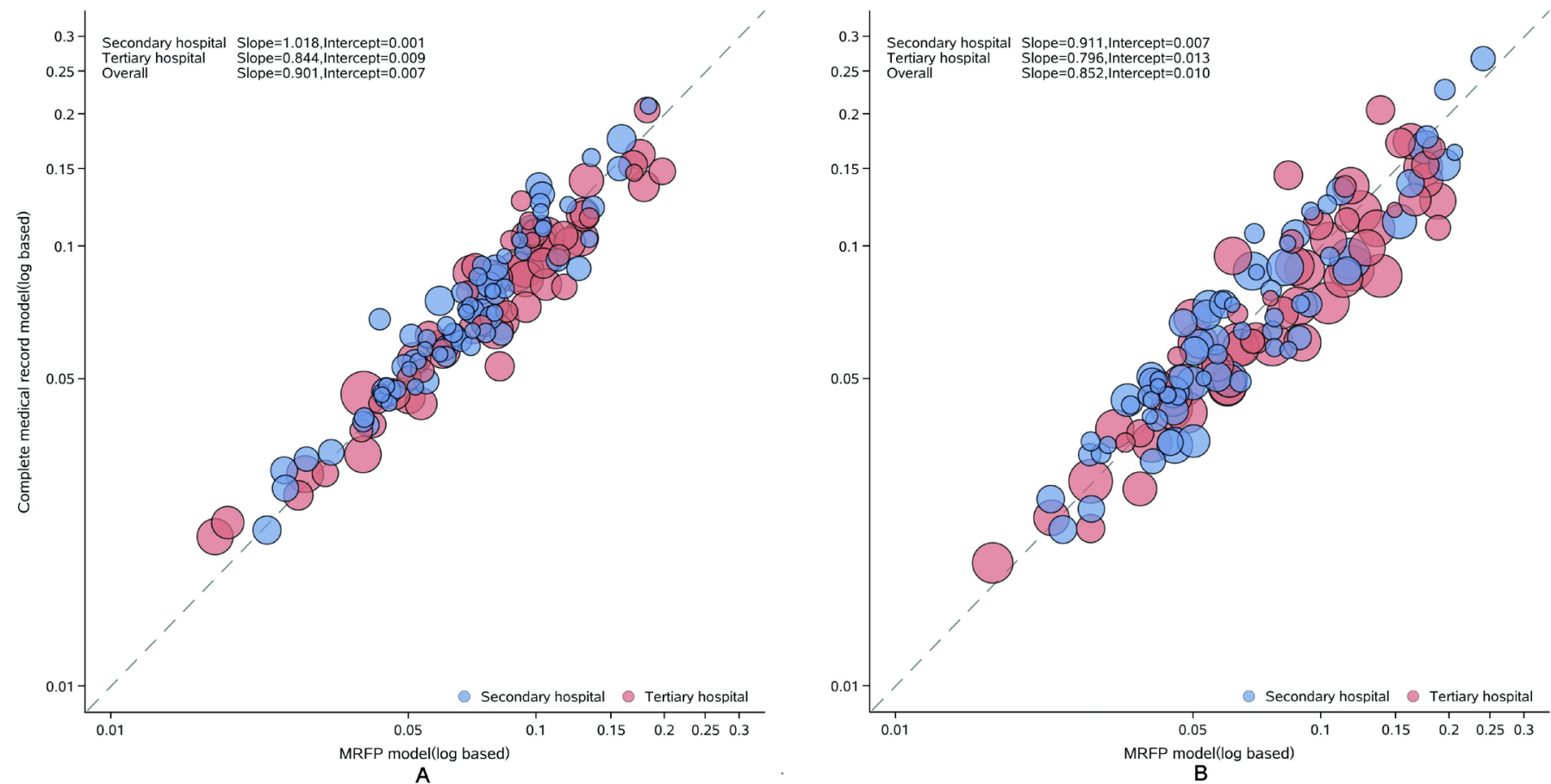

Figure 3 Correlation of risk standardised mortality rate estimated by MRFP model and complete medical record model. (A) Modelling cohort and (B) validation cohort. MRFP, medical record front page.

The predictors included previously were applied to the validation cohort to reconstruct the model, which showed that the effect direction and size were still similar (figure 2A). In the validation cohort, the AUC was 0.79, with observed mortality rate ranging from $1.00 \%$ to $29.72 \%$, and the slope and intercept of the calibration curve was 0.93 and 0.005 (online supplemental figure S2 and table 2).

Using the same method, a complete medical record model was built, in which a total of 13 risk factors were included (figure 2B). The AUC of the model was 0.79, and observed mortality rate ranged from $0.51 \%$ in the lowest decile to $27.96 \%$ in the highest decile. The slope of the calibration curve was 0.94 , and the intercept was 0.004 (online supplemental figure S2 and table 2). Similar with the MRFP model, the complete medical record model had good discrimination and calibration, as well as relatively stable coefficients when validated among the validation cohort (figure 2B, online supplemental figure S2 and table 2). Additional analysis showed that both our two patient risk prediction model had better AUC (all $\mathrm{p}$ value $<0.001$ ) and positive IDI among development and validation cohorts compared with the GRACE prediction model (online supplemental appendix A).

\section{Development and comparison of hospital-level model}

A total of 8269 patients (137 hospitals, 73 secondary hospitals and 64 tertiary hospitals) from modelling cohort and 9583 patients (132 hospitals, 71 secondary hospitals and 61 tertiary hospitals) from validation cohort were included in estimating the hospital-level HGLMs.

In the modelling cohort, the median hospital-level RSMR was $7.4 \%$ (IQR: $5.2 \%-10.1 \%$ ). The median of absolute difference between the RSMR predicted by the complete medical record data and MRFP data was $0.08 \%$ (IQR: $-0.67 \%$ to $0.53 \%$ ), and the 10 th and 90 th percentiles were $-1.8 \%$ and $1.6 \%$, with no statistical significance $(\mathrm{p}=0.499)$. In the validation cohort, the median RSMR was 6.4\% (IQR: 4.5\%-10.4\%), and the median of absolute difference was $0.05 \%$, with 10th and 90th percentiles of $-2.8 \%$ and $1.9 \%$ (online supplemental figure S3). For the regression model comparing the RSMR between the MRFP data and complete medical record data, the slope (intercept) was $0.90(0.007)$ in the modelling cohort, while $0.85(0.010)$ in the validation cohort (figure 3). The correlations among secondary hospitals were better than among tertiary hospitals.

\section{DISCUSSION}

This study developed patient- and hospital- level MRFP models of in-hospital mortality of AMI, and took into account the patient case-mix in the hospital-level disparity analysis. These models based on MRFP data showed good discrimination and calibration capability, as well as similar risk prediction effect in comparison with the model based on complete medical record data, which proved that MRFP data could be suitable for risk adjustment in hospital performance measurement in China.

To our knowledge, the current study extended literatures in several ways. First, this is the first in-hospital mortality risk model based only on MRFP data in China. Currently, in China, it is still difficult to obtain detailed complete medical records data nationwide for quality monitoring due to the fragmentation in development and deployment of Hospital Information Systems and Electronic Medical Record Systems. 
In the USA, which faces similar challenges, several risk models have been developed using concise administrative claims data and successfully applied as substitute of complete medical record models. ${ }^{89}$ The key value of this model is to demonstrate how MRFP data from HQMS can serve as a solution for national quality assessment, rather than to identify coefficients of specific risk characteristics.

Second, the methods we chose for model development had advantages in managing patient case-mix and obtaining the hospital-level risk-standardised rates. We first selected an array of patient characteristics which influenced their risk profile significantly using backward logistic regression, and confirmed the stability of this array in the validation cohort. Then, we established a HGLM with these characteristics as covariates, which could take the correlation of patients in the same hospital into account and avoid underestimating the SE of other risk factors, ${ }^{18} 19$ thus it reflected the nature that patients were clustered within individual hospitals. This method has been well-tested in previous studies on hospitallevel comparisons. ${ }^{7-9}$

Third, the MRFP model was robust when we repeated the analysis in validation cohorts, and more importantly, comparable to the model derived from complete medical records data with similar validities. Even though there is no real golden standard of risk standardisation, medical record data enable the most complete characteristics of patients' demographic and clinical profile. The China PEACE retrospective study provided a unique opportunity to compare the MRFP model against the complete medical record model, because scanning copies of sampled medical records were collected and detailed information on patient characteristics had been centrally extracted from the front page and all other parts of medical records.

The feasibility of MRFP model has significant policy implications for China, as the government emphasised the importance of hospital performance monitoring. ${ }^{20}$ China needs a nationwide data platform, which supports timely, accurate and sustainable outcome measurement, since the outcomes of care such as mortality provide a global assessment of quality and have the most relevance to patients. However, outcome measurement is challenging because of variation among hospitals in patients' risk profile; meanwhile, extracting data from electronic medical records is infeasible in most hospitals. Our study first proved that concise MRFP data that are available in the HQMS can sufficiently reflect patients' risk profile, which makes it suitable to generate risk-standardised mortality rates at hospital level. Thus, this existing platform covering 1800 (73\%) tertiary hospitals and 2300 (26\%) secondary hospitals can serve as a base for national hospital performance measurement similar to the US Centres for Medicare \& Medicaid Services' use of administrative claims data. ${ }^{19}{ }^{20}$ Moreover, some challenges should to be addressed. First, the quality of MRFP data across hospitals, particularly the completeness of comorbidity documentation and accuracy of diagnosis coding in diagnosis, needs to be improved. ${ }^{21}$ Second, for chronic conditions with low in-hospital mortality rates, data on postdischarge outcomes (eg, 30-day readmission rates) data need to be obtained from clinical registries, insurance claims and other sources.

\section{Limitations of the study}

There are some limitations in this study. First, weaker correlation in tertiary hospitals between RSMRs generated from the two risk models indicated a relatively poorer performance of current MRFP model applied in tertiary hospitals. However, this could be improved if the model development and disparity assessment were conducted within subgroups of hospitals separately. Second, although this study was based on nationally representative cohorts with model development and validation using data from different years, external validations that include more diverse hospitals will be needed in the future.

\section{Conclusion}

In conclusion, the MRFP model of in-hospital mortality supported that HQMS data could act as reasonable substitute for complete medical record data in risk adjustment between hospitals across the nation. The lessons from AMI treatment could serve as a model to nationwide assessment on quality of care in other clinical fields.

\section{Author affiliations}

${ }^{1}$ National Clinical Research Center of Cardiovascular Diseases, NHC Key Laboratory of Clinical Research for Cardiovascular Medications, State Key Laboratory of Cardiovascular Disease, Fuwai Hospital, Chinese Academy of Medical Sciences and Peking Union Medical College, National Center for Cardiovascular Diseases, Beijing, China

${ }^{2}$ Center for Outcomes Research and Evaluation, Yale-New Haven Health System, New Haven, Connecticut, USA

${ }^{3}$ Section of Cardiovascular Medicine, Yale School of Medicine, New Haven, Connecticut, USA

Acknowledgements We appreciate the multiple contributions made by study teams at National Clinical Research Center for Cardiovascular Diseases and Yale-New Haven Hospital Center for Outcomes Research and Evaluation in study design and operations, particularly the data collection by Yi Pi, Jiamin Liu, Wuhanbilige Hundei, Haibo Zhang, Lihua Zhang, Wenchi Guan, Xiaofang Yan, Yuan Yu, Xiqian Huo, Xin Zheng, and Yuanlin Guo. We appreciate the editing by Aoxi Tian and Xingyi Zhang. We are grateful for the support provided by the Chinese government.

Collaborators China PEACE-Retrospective AMI Study Site Investigators: Shiping Weng, Shuying Xie, Lirong Wu, Jiulin Chen, Tianfa Li, Jun Wang, Qin Yu, Xiaofei Li, Zhong Li, Shiguo Hao, Yuzhen Zhang, Xuemei Wu, Yachen Zhang, Zhifeng Liu, Zhongxin Wang, Hao Jia, Bayin Bate, Badeng Qiqige, Xiang Jin, Ting Cai, Fengqin Liu, Dayong Xu, Xuejin He, Shui Yang, Chun Yuan, Jiping Wang, Lihua Gu, Lin Li, Shijiao Chen, Yongchao Zhi, Lili Sun, Shengcheng Zhou, Lingjiao Jin, Yong Leng, Liangchuan Zhang, Tianyun Deng, Yuanjin Wang, Wenhua Zhang, Xinmin Ma, Weimin Li, Liang Lu, Xuan Ge, Xiaoping Wu, Yanming He, Fanju Meng, Jia Li, Dexi Liao, Guangyong Liu, Wen Qin, Wen Long, Xiangwen Chen, Baohong Zhang, Yonghou Yin, Bin Tian, Yong Yi, Chaoyong Wu, Baogi Liu, Zhihui Zhao, Haiming Li, Yansong Guo, Xinjing Chen, Liquan Xiang, Lin Ning, Mei Chen, Xin Jin, Guiling Li, Xiuqi Li, Xing'an Wu, Congjun Tan, Mingfang Feng, Meili Wang, Liangfa Wen, Xiang Fu, Qunxing Xie, Wei Zhang, Yanni Zhuang, Hua Lu, Jiaqian Lu, Yu Huang, Yin Zhou, Qiuling Hu, Chunhui Xiao, Xiaoli Hu, Yongshuan Wu, Qiuli Wang, Youlin Xu, Xuefei Yu, Chuanyu Gao, Jianhong Zhang, You Zhang, Wentang Niu, Xiaolei Ma, Yong Wang, Xiaowen Pan, Yanlong Liu, Lifu Miao, Yanping Yin, Zhiying Zhang, Shutang Feng, Aiping Wang, Jiangli Zhang, Feipeng Li, Hong Wang, Lijun Yu, Xinxin Zhao, Yuansheng Shen, Zhiming Li, Lizhen He, Zhiyi Rong, Wei Luo, Xueqiao Wang, Rongjun Wan, Jianglin Tang, Guanghan Wu, Jie Wu, Bin Xu, Qing Huang, Xiaohe Wu, Sang Ge, Pian Pu, Pingcuo Duoji, Hui Dai, Yuming Du, Wei Guo, Jianping Shi, Peihua Zhao, Jingsheng Sun, Hongxiang Li, Wen Liang, Zhiwen Dong, Zhenhai Zhao, Xin Li, Qin Xu, Yaofeng Yuan, Zhirong Li, Jinbo Gao, Qiu'e Guo, Ruiqing Zhao, Guangjun Song, Lize Wang, Haiyun Song, Jinwen He, Jinming He, Keyong Shang, Changjiang Liu, Kuituan Xi, 
Rihui Liu, Peng Guo, Chaoyang Guo, Xiangjun Liu, Rujun Zhao, Zeyong Yu, Wenzhou Li, Xudong Jing, Huanling Wang, Xiyuan Zhao, Chao Zhang, Long Chen, Meifa Wei, Yan Liu, Shengde Chen, Kaihong Chen, Yong Fang, Ying Liao, Junli Wang, Tianyu Liu, Suzhe Cheng, Yunke Zhou, Xiaoxia Niu, Huifang Cao, Zebin Feng, Min Feng, Feilong Duan, Haiming Yi, Yuanxun Xu, Anran Guo, Xianshun Zhou, Hongzhuan Cai, Peng Zheng, Gaofeng Guo, Xiang Li, Minwu Bao, Yuhong Liu, Shaoliang Chen, Haibo Jia, Hongjuan Peng, Duanping Dai, Shaoxiong Hong, Song Chen, Dongya Zhang, Ying Wang, Yudong Li, Jianbu Gao, Shouzhong Yang, Junhu An, Chenyang Shen, Yunfeng Liu, Chun Wu, Huan Qu, Saiyong Chen, Yuhui Lin, Dehai Jiao, Manhong Wang, Qiu Wang, Yingliang Xue, Ruijun Zhang, Cheng Yuan, Lei Wu, Jianqing Zhang, Chunmei Wei, Yanmei Shen, Hehua Zhang, Hongmei Pan, Yong Gao, Xiaowen Ma, Yanli Liang, Tianbiao Wang, Daguo Zhao, Xiaoming Tu, Zhenyan Gao, Fangning Wang, Qiang Yang, Xiaoping Kang, Jianbin Fang, Dongmei Liu, Chengning Shen, Mengfei Li, Yingmin Guan, Wenfeng Wang, Ting Xiao, Qian Wang, Fengyun Jiang, Kaiyou Wu, Songguo Wang, Xujie Fu, Shu Zhang, Lifang Gao, Min Zhang, Kai Fu, Xiaojing Duan, Rui Xiao, Ruixia Wu, Bin Li, Hongtu Zhang, Yuerong Ma, Zhonghui Cao, Zhansheng $\mathrm{Ba}$, Wanhai Fu, Jianjun Jiang, Yafei Mi, Weiwei Zhou, Feng Sun, Qi Zhang, Shiyu Zheng, Jing Zhang, Yang Zhong, Fangjiang Li, Xiaoyuan Wang, Pingshuan Dong, Laijing Du, Wei Liu, Zhaofa He, Meihua Jin, Ting Jiang, Zhuoyan Chen, Manli Cheng, Yuqiang Ji, Youhua Zhou, Jvyuan Li, Yizhi Pan, Jian Liu, Tianxun Wang, Ping Yang, Guiyu Huang, Jianjun Pan, Qingliang Cai, Qianying Wang, Yuanming Yi, Xuelian Deng, Wenhua Chen, RongCai, Bing Zhang, Bo Yu, Yousheng Xu, Zhengqiu Wang, Jun Shu, Ge Zhang, Kai Li, Guang Ma, Puxia Suo, Aimin Zhang, Yongfen Kang, Zheng Wan, Yuemin Sun, Bo Bian, Xuejun Hu, Dawa Ciren, Guojiong Jia, Jieli Pan, Guofu Li, Hongliang Zhang, Longliang Zhan, Junping Fang, Xinli Yu, Dacheng Wang, Dajun Liu, Xinhong Cao, Yi Tian, Haisheng Zhu, Wanchuan Liu, Zhaohai Zhou, Lei Shi, Wuwang Fang, Manxin Chen, Fuqin Han, Jianye Fu, Yunmei Wang, Binglu Liu, Yanliang Zhang, Xiupin Yuan, Qingfei Lin, Yun Chen, Yuliang Zhu, Zhiqiang Cai, Xingping Li, Lirong Ao, Shubing Wu, Hui Zhang, Fusheng Zhao, Guangming Yang, Renfei Liu, Wenwei Ai, Jianbao Chang, Haijie Zhao, Qijun Ran, Xuan Ma, Shijun Jiang, Xiaochun Shu, Zhiru Peng, Yan Han, Jianbin Wang, Li Yang, Yu Shen, Xingcun Shang, Haifeng Wang, Hongyan Li, Zhisong Liao, Yang Cao, Xiaoping Gao, Meiying Cai, Lining You, Xuexin Li, Shuqin Li, Yingjia Li, Jianxun Yang, Song Ai, Jianfei Ma, Lailin Deng, Keyu Wang, Shitang Gao, Jian Guan, Banghua He, Youyi Lu, Weirong Yang, Hong Li, Zhizhong Zhang, Xiaohong Chi, Ru Duan and Guangli Wang.

Contributors XL contributed to the conception or design of the work. CW, DZ and $\mathrm{XB}$ contributed to the acquisition of data for the work. $\mathrm{CW}$ and $\mathrm{XB}$ contributed to the analysis of data for the work. CW, DZ, TZ and XL contributed to the interpretation of data for the work. CW and DZ drafted the manuscript. TZ, YW, ZL, GH and $\mathrm{XL}$ critically revised the manuscript. All gave final approval and agree to be accountable for all aspects of work ensuring integrity and accuracy.

Funding This work was supported by the National Key Research and Development Program (2017YFC1310803) from the Ministry of Science and Technology of China, the Chinese Academy of Medical Sciences Innovation Fund for Medical Science (2017-I2M-2-002) the 111 Project from the Ministry of Education of China (B16005) and the National Key Technology R\&D Program (2015BAl12B01) from the Ministry of Science and Technology of China.

Disclaimer The sponsors had no role in the conduct of the study; in the collection, management, analysis and interpretation of the data; or in the preparation or approval of the manuscript.

Competing interests None declared.

Patient consent for publication Not required.

Ethics approval The Ethics Committee at the National Center for Cardiovascular Diseases approved the study (2012-377; 2016-769). All collaborating hospitals either accepted central ethics approval or obtained local ethics approval by their ethics committees.

Provenance and peer review Not commissioned; externally peer reviewed.

Data availability statement № additional data available.

Supplemental material This content has been supplied by the author(s). It has not been vetted by BMJ Publishing Group Limited (BMJ) and may not have been peer-reviewed. Any opinions or recommendations discussed are solely those of the author(s) and are not endorsed by BMJ. BMJ disclaims all liability and responsibility arising from any reliance placed on the content. Where the content includes any translated material, BMJ does not warrant the accuracy and reliability of the translations (including but not limited to local regulations, clinical guidelines, terminology, drug names and drug dosages), and is not responsible for any error and/or omissions arising from translation and adaptation or otherwise.
Open access This is an open access article distributed in accordance with the Creative Commons Attribution Non Commercial (CC BY-NC 4.0) license, which permits others to distribute, remix, adapt, build upon this work non-commercially, and license their derivative works on different terms, provided the original work is properly cited, appropriate credit is given, any changes made indicated, and the use is non-commercial. See: http://creativecommons.org/licenses/by-nc/4.0/.

ORCID iD

Xi Li http://orcid.org/0000-0003-2249-688X

\section{REFERENCES}

1 General Office of the State Council of the People's Republic of China. Opinions of the general office of the state council on strengthening the performance evaluation of public hospitals, 2019.

2 National Health Commission. Measures for medical quality management. secondary measures for medical quality management, 2016. Available: $\mathrm{http} / / / w w w . n h c . g o v . c n / f z s / s 3576 / 201610 / a e 125 f 28$ eef24ca7aac57c8ec530c6d2.shtml

$3 \mathrm{Li}$, Krumholz HM. What does it take to improve nationwide healthcare quality in China? BMJ Qual Saf 2019;379:bmjqs-2019-009839.

4 Jiang L, Krumholz HM, Li X, et al. Achieving best outcomes for patients with cardiovascular disease in China by enhancing the quality of medical care and establishing a learning health-care system. Lancet 2015;386:1493-505.

5 Department of Medical Management of the Ministry of Health. Notice on the pilot project of direct reporting of medical service supervision information network, 2011

6 Bureau of Medical Administration of National Health Commission. Hospital quality monitoring system. secondary Hospital quality monitoring system. Available: https://www.hqms.org.cn/login.jsp

7 Granger CB, Goldberg RJ, Dabbous O, et al. Predictors of hospital mortality in the global registry of acute coronary events. Arch Intern Med 2003;163:2345-53.

8 Krumholz HM, Wang Y, Mattera JA, et al. An administrative claims model suitable for profiling hospital performance based on 30-day mortality rates among patients with an acute myocardial infarction. Circulation 2006;113:1683-92.

9 Krumholz HM, Chen J, Wang Y, et al. Comparing AMI mortality among hospitals in patients 65 years of age and older: evaluating methods of risk adjustment. Circulation 1999;99:2986-92.

$10 \mathrm{Li} \mathrm{X}$, Murugiah K, Li J. Urban-rural comparisons in hospital admission, treatments, and outcomes for ST-Segment-Elevation myocardial infarction in China from 2001 to 2011. Circulation 2017;10:e003905.

11 Dharmarajan K, Li J, Li X, et al. The China patient-centered evaluative assessment of cardiac events (China peace) retrospective study of acute myocardial infarction: study design. Circ Cardiovasc Qual Outcomes 2013;6:732-40.

12 Antman EM, Cohen M, Bernink PJ, et al. The TIMI risk score for unstable angina/non-ST elevation MI: a method for prognostication and therapeutic decision making. JAMA 2000;284:835-42.

13 Morrow DA, Antman EM, Charlesworth A, et al. TIMI risk score for ST-elevation myocardial infarction: a convenient, bedside, clinical score for risk assessment at presentation: an intravenous nPA for treatment of infarcting myocardium early II trial substudy. Circulation 2000;102:2031-7.

14 Chin CT, Chen AY, Wang TY, et al. Risk adjustment for in-hospital mortality of contemporary patients with acute myocardial infarction: the acute coronary treatment and intervention outcomes network (ACTION) registry-get with the guidelines (GWTG) acute myocardial infarction mortality model and risk score. Am Heart $J$ 2011;161:113-22.

15 Morrow DA, Antman EM, Giugliano RP, et al. A simple risk index for rapid initial triage of patients with ST-elevation myocardial infarction: an InTIME II substudy. Lancet 2001;358:1571-5.

16 Lee KL, Woodlief LH, Topol EJ, et al. Predictors of 30-day mortality in the era of reperfusion for acute myocardial infarction. Results from an international trial of 41,021 patients. GUSTO-I Investigators. Circulation 1995;91:1659-68.

17 Normand ST, Glickman ME, Sharma RG, et al. Using admission characteristics to predict short-term mortality from myocardial infarction in elderly patients. Results from the cooperative cardiovascular project. JAMA 1996;275:1322-8.

18 Shahian DM, Torchiana DF, Shemin RJ, et al. Massachusetts cardiac surgery report card: implications of statistical methodology. Ann Thorac Surg 2005;80:2106-13. 
19 Austin PC, Tu JV, Alter DA. Comparing hierarchical modeling with traditional logistic regression analysis among patients hospitalized with acute myocardial infarction: should we be analyzing cardiovascular outcomes data differently? Am Heart $J$ 2003;145:27-35

20 Xinhua News Agency. Interpretation on "the guiding opinions on the establishment of modern hospital management system" by the head of the Medical Reform Office of the State Council. Secondary
Interpretation on "the guiding opinions on the establishment of modern hospital management system" by the head of the Medical Reform Office of the State Council Jul 26 2017. Available: http:// www.gov.cn/xinwen/2017-07/26/content_5213541.htm

21 Wang $Y$. The challenge and solution for the front page of inpatient medical record based on DRGs. Chinese $J$ Hospital Admin 2015;31:831-3. 